\title{
Crestal sinus lift using an implant with an internal L-shaped channel: One-year after loading results from a prospective cohort study
}

\author{
Erta Xhanari2, Marco Tallarico'1, Silvio Mario Meloni ${ }^{3}$ \\ ${ }^{1}$ Private practice, Rome, Italy; ${ }^{2}$ Private practice, Tirana, Albania; ${ }^{3}$ Adjunctive prof., University of Sassari, Italy.
}

Introduction and aim: Maxillary sinus floor elevation using a lateral approach implies execution of a large mucosal periosteal flap that inevitably affects post-operative recovery and the additional cost of the augmentation procedure. Sinus membrane perforations, nose bleeding, post-operative pain, swelling, hematoma, and possible sinus infection are possible complications. The aim of the resent study was to evaluate the clinical and radiographic outcomes of a one-stage crestal sinus elevation procedure using a self-tapping endosseous implant system (iRaise, Maxillent, Herzliya, Israel) developed for sinus augmentation, one year after loading.

Materials and Methods: Patients needing restoration in the posterior maxilla with a residual alveolar crest of 3 to $8 \mathrm{~mm}$ in height and $5 \mathrm{~mm}$ in width distal to the canine as measured on CBCT scan were treated using the iRaise sinus lift system. Outcome measures were: implant and prosthetic failures, any complications, increased bone height (iBH), marginal bone loss (MBL), implant stability quotient (ISQ), radiographic tissue remodeling patterns using the sinus grafting remodeling index (SGRI), volumetric measurements of sinus graft, patient self-reported post-surgical swelling, consumption of pain medication and histological analysis.
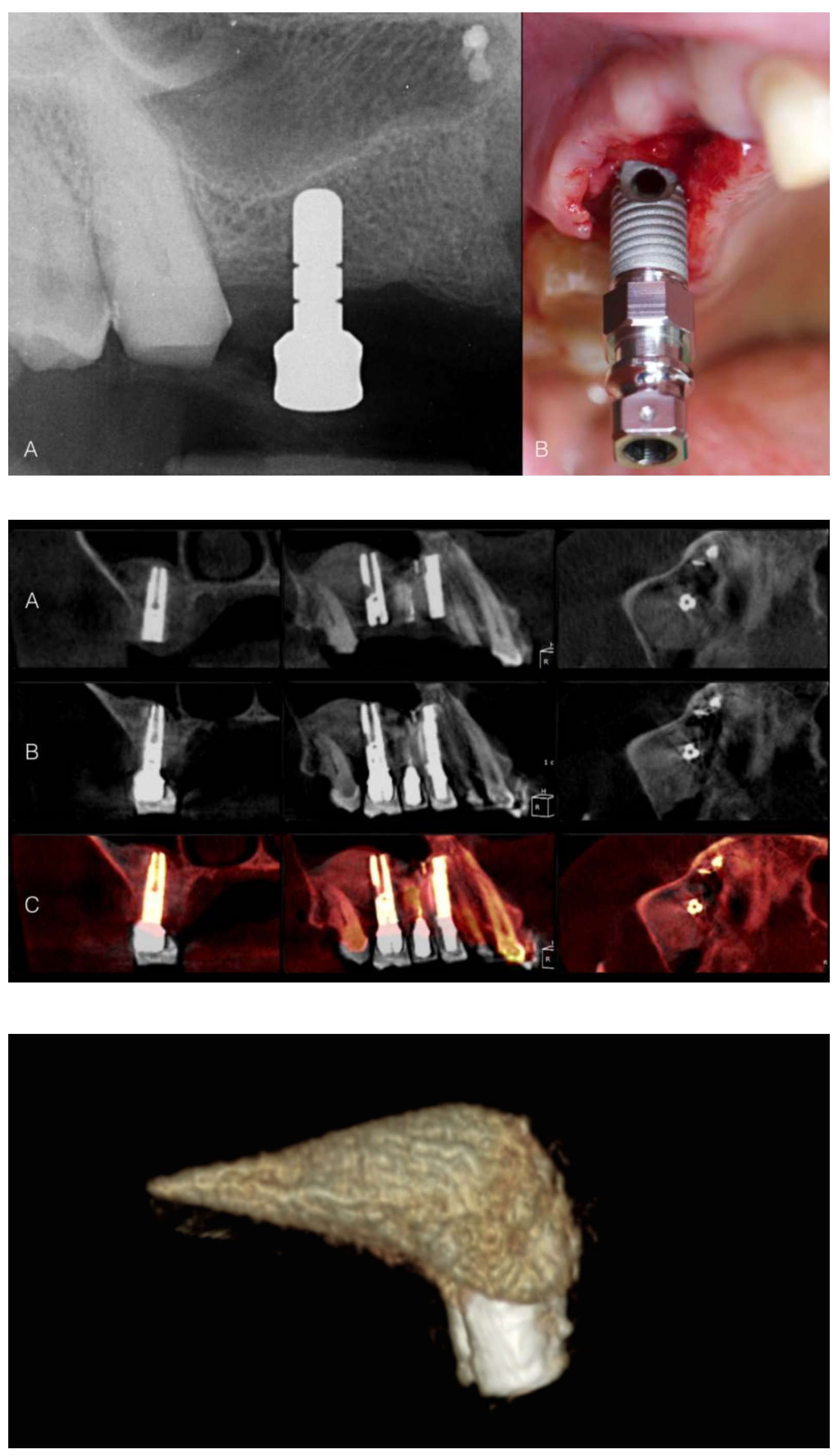

Results: A total of 30 consecutive participants with a mean age of 54.2 \pm 9.4 years underwent a transcrestal elevation of the sinus membrane, insertion of bone graft, and implant placement. A total of 50 implants were placed (30 iRaise system implants and 20 adjunctive iSure implants, Maxillent). The mean follow-up was $15.8 \pm 2.1$ months after implant loading. One patient dropped out at the 1-year after loading follow-up examination. No implants and no prostheses failed during the entire follow-up. One patient experienced a small membrane tear. Before implant insertion, the mean residual alveolar ridge height was $4.64 \pm 0.86 \mathrm{~mm}$ (range: $3.4-6.4 \mathrm{~mm}$; 95\% CI: 4.39-5.01 mm). One year after loading, the bone height was $16.86 \pm 3.13 \mathrm{~mm}(95 \% \mathrm{CI}$ 15.83-18.07 mm). At the 1-year after loading follow-up, the mean MBL was $0.19 \pm 1.05 \mathrm{~mm}(95 \%$ CI $0.02-0.78 \mathrm{~mm})$. The mean ISQ at implant placement was $65.2 \pm 5.4$ (95\% CI 63.6-67.4) and increased during the heal- ing period reaching the mean value of $73.6 \pm 3.7(95 \%$ CI 73.1-75.9; range 62-79). The difference was statistically significant $(8.4 \pm 5.3 ; 95 \%$ CI $5.9-39.7 ; P=0.0000)$. One year after loading, SGRI score was evaluated in 23 implants. Overall, the mean SGRI value was $2.29 \pm 2.41 \mathrm{~mm}$ (95\% CI $1.22-2.98 \mathrm{~mm})$. Bone volume at implant placement was $2.41 \pm 0.25 \mathrm{CC}(95 \%$ CI $2.22-2.48 \mathrm{CC})$. During the 6-month, submerged healing period, a slight bone contraction of $11.3 \%$ were observed. $(2.13 \pm 0.24$ CC;95\% CI $2.02-$ 2.26; difference $=0.27 \pm 0.25 \mathrm{CC} ; 95 \%$ CI $0.10-0.36 ; P=0.0011)$. At the first year post-loading period, the bone graft remained stable (2.11 $\pm 0.22 \mathrm{CC} ; 95 \%$ CI $2.02-2.24)$. The difference was not statistically significant $(0.02 \pm 0.07 \mathrm{CC} ; 95 \%$ CI $0.01-0.04 ; P=0.2166)$. From the patient's point of view, the mean pain value was $0.52 \pm 0.74$ (range 0 3 ); mean swelling value was $0.27 \pm 0.52$ (range $0-2$ ); and the mean consumption of analgesic was $0.87 \pm 4.94$ tablets (range 0-4) 3 days after surgery. Morphological and histomorphometric analyses showed that all the samples had a normal structure without inflammatory infiltrate, six months after healing. The following fractions (\%) were found: bone (immature bone + mature bone): $44.07 \pm 4.91$; residual biomaterial: $23.98 \pm 2.64$; medullary spaces: $31.95 \pm 3.16$.

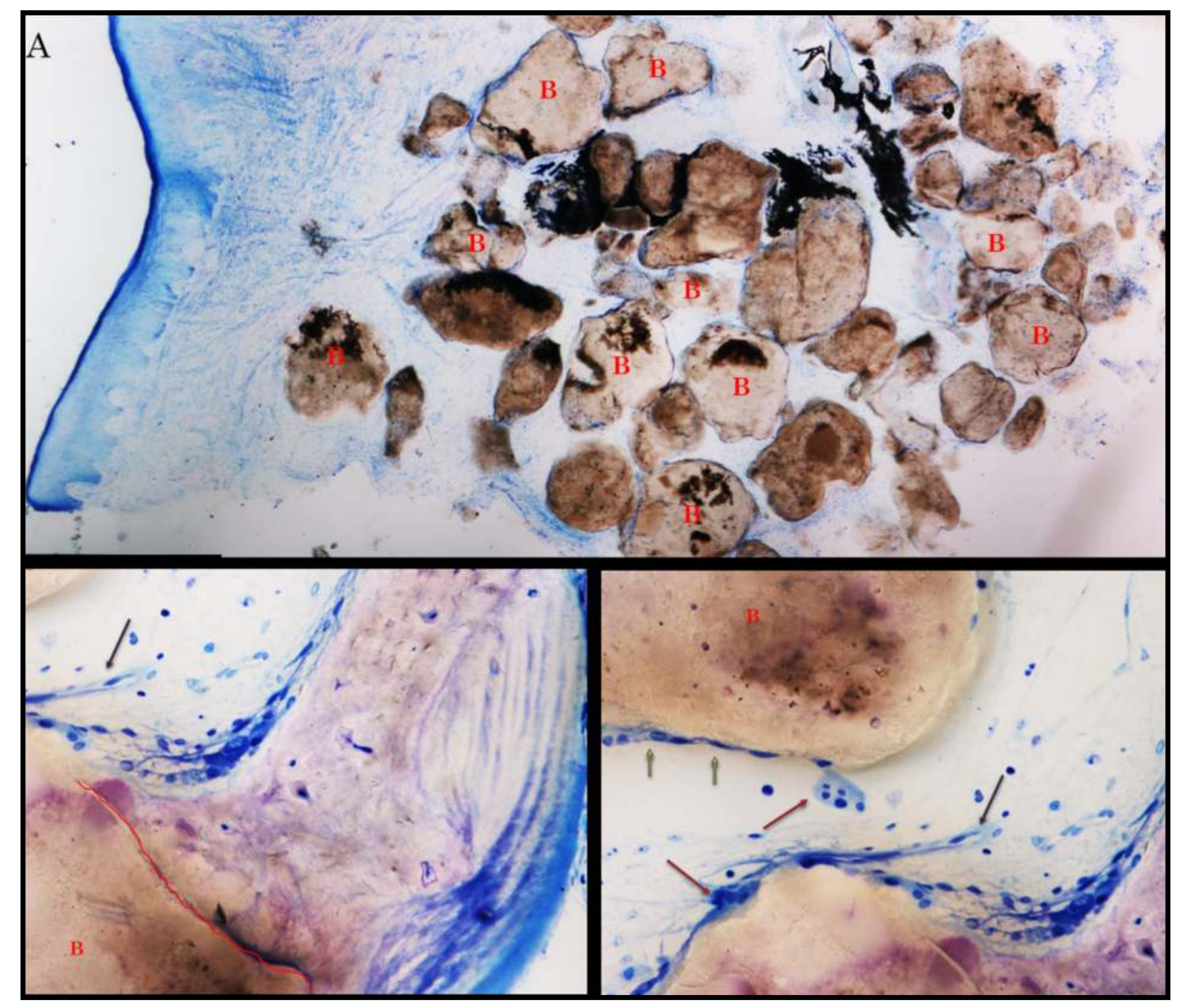

Conclusions: Sinus floor augmentation can be successfully accomplished with a transcrestal approach using a dedicated implant system. A physiologic contraction of $11.3 \%$ of the original volume of the bone graft was experienced during the first 6 months of healing; afterwards, no additional graft volume reduction was observed. Longterm clinical studies are needed to con rm these preliminary results. 